\title{
Status recognition for fused deposition modeling manufactured parts based on acoustic emission
}

\author{
Feng $\mathrm{Li}^{1}$, Zhonghua $\mathrm{Yu}^{1}$, Xuanwei Shen ${ }^{1}$ and Hao Zhang ${ }^{1}$ \\ ${ }^{1}$ The State Key Laboratory of Fluid Power Transmission and Control, College of Mechanical Engineering, Zhejiang University, Hangzhou, \\ 310027, China
}

\begin{abstract}
Fused deposition modelling (FDM), as one technology of additive manufacturing, fabricates parts always with curl and looseness defects which restrict its development to a great extent. In this paper, a method based on acoustic emission (AE) was proposed to recognise the status of the manufactured part in FDM process. Experiments were carried out to acquire the AE signal when the printing part was respectively in normal, looseness and curl state. The ensemble empirical mode decomposition (EEMD) was employed to the process of feature extraction and both the methods of Hidden-semi Markov model (HSMM) and support vector machine(SVM) were applied to recognise the three states of the normal, looseness and curl. The results reveal that the combination of EEMD and HSMM makes it more accurate to recognize these three states.
\end{abstract}

\section{Introduction}

Additive manufacturing (AM) technology have been gaining more and more popularity for its ability in fabricating complex parts without any tooling requirement and human interface. The quality of $\mathrm{AM}$ is still poorer than which of traditional subtractive manufacturing. Researchers have made many efforts to improve the quality of parts printed by AM. Filling path was optimized by Jin et al [1]. In their paper, a parallelbased path was generated where the sequence of the filling area was optimized and after that the overfilling and under filling phenomena were relieved. Printing parameters make a complex effect on the fabricated parts. Parameters optimization were conducted in $[2,3]$ where experiments were carried out and impacts of process parameters like layer thickness and extrusion velocity on quality of printed parts which were compressive stress and dimensional error here were respectively explored. The specific impacts of the process parameters on the quality of AM fabricated parts have not been understood yet considering the complexity it works. Mechanical properties like fatigue and sliding wear were researched in $[4,5]$. Ravari et al. [6] constructed the beam and solid model and then, carried out the experiment to study the mechanical behaviour of the printed part with lattice structures. According to the authors, solid model predicts a lower mechanical stress-strain curve than that by the beam model and at least ten and twelve intervals should be considered for each strut to achieve reasonable elastic modulus and collapse stress in the process of numerical simulation.
Temperature monitoring was carried out by Abdelrahman [7] in selective laser sintering (SLS) process, where high resolution infrared thermal imaging was used before, during, and after laser scanning. A relationship between the temperature and the geometry of the part, the layer cross-sectional area, and the presence or absence of an underlying solid layer was shown. Heterogeneous sensors including accelerometers, thermocouples, an infrared (IR) temperature sensor, and a real-time video borescope were employed to monitoring the platform vibration, extruder vibration, platform temperature, extruder temperature, melt-pool temperature and real-time condition [8]. A predictive model was built successfully to give an instruction about the failure modes. Other researches like [9] aimed at the monitoring of process variables as well.

FDM is one technology of AM and has been widely used for its low cost, easy operation and little pollution. During FDM, the material filament was heated to a semimolten state and then, extruded through the printing nozzle in a prescribed path onto a platform or a deposited layer. After a layer was fabricated, the printing nozzle moves up or the platform moves down in a layer's height and this printing process continued until the whole part is completed. The deposited material cools, solidifies and bonds with the neighbouring material. This heating and rapid cooling cycles of the work materials will aggravate non-uniform thermal gradients and cause stress build-up that consequently results in defects. The monitoring approaches carried out in FDM process can be divided into the destructive testing and the non-destructive testing. Fibre Bragg grating or thermocouple [11,12] based investigations of temperature, residual stresses and 
residual strains are typical destructive testing which were embedded into the fabricated parts in FDM process. Understanding the development process of temperature, residual stresses and residual strains contributes to choosing the suitable parameters to avoid the defects appearing. The non-destructive testing method is mainly based on the Machine Vision (MV). Yi et al. [13] combined the MV and Statistical Process Control (SPC) to detect defects in FDM. The results proved the proposed method could detect defects during manufacturing process successfully. Although the method still need a certain time to make decisions. Ultrasound inspection is another available way to monitor the defects in FDM [14-16]. However, the application of it has been restricted to the delamination inspection and not mature yet.

Considering that the availability of $\mathrm{AE}$ sensor in detecting stress wave when the structure of the printing part changed, this paper presented a monitoring method based on AE. Data pre-processing was implemented to normalize and de-noise the acquired AE signals. Further, EEMD was applied to extract features of the signals in different states. Both the HSMM and SVM were employed to recognise the three states and finally the recognition results were compared and discussed. In the remainder of this paper, the relative methodologies are introduced in Section 2. In Section 3, experimental setup and procedures are presented. We give the experiment results and discussions in Section 4.

\section{Methodologies}

\subsection{Feature extraction method of ensemble empirical mode decomposition}

EEMD was proposed by $\mathrm{Wu}$ and Huang [17] and it is a method developed on the basis of empirical mode decomposition (EMD) which is suitable for the handling of nonlinear and non-stationary signals. EEMD was proposed to overcome the major drawback of EMD called mode mixing problem [17] by artificially adding a series of Gaussian white noise in the decomposition process of the target signal. It has been widely used in the fault diagnosis of the rotating machinery [18-22].

The title is set in bold 14-point Arial, flush left, unjustified. The first letter of the title should be capitalised with the rest in lower case. You should leave $35 \mathrm{~mm}$ of space above the title and $6 \mathrm{~mm}$ after the title.

\subsection{Pattern recognition method of hidden semi- Markov model and support vector machine}

\subsubsection{Hidden semi-Markov model}

HSMM [23] is an improved method on the basis of hidden Markov model (HMM) which is a simple dynamic Bayesian network and makes inference by calculating the value of likelihood of being in certain discrete "hidden states". Each state of HSMM has variable duration and a number of observations being produced while in the state.
Thus, a wider range it has been applied. Dong and $\mathrm{He}$ improved the precision of fault diagnosis by using HSMM [24] and states diagnosis and prediction was made as well. Human activities of daily living (ADL) was learned and recognized by Duong et al. in [25]. A statistical speech synthesis system based on HSMM was proposed [26] considering the inconsistency HMM made.

\subsubsection{Support vector machine}

SVM was proposed by Vapnik [27] and has been widely used in the fault diagnosis of traditional manufacturing machine [28,29], monitoring of the tool wear [30] and abnormality detection on-line [31].The voting strategy is adopted[32] to determine the classes of the input data.

SVM is a supervised algorithm. So it is necessary to divide the samples to the training data and test data. The generalization ability of SVM is depended on the chosen kernel function to a great extent. Furthermore, SVM is adaptive to the two-class problem and it needs to construct many SVMs when handling multi-class problem.

\subsection{Model performance measurement based on F-score}

Precision ratio and recall ratio are two indexes which can response the constructed model performance. The samples are divided to categories of true positive, false positive, true negative and false negative for a two-class problem. Let $T P, F P, T N$, and $F N$ respectively represent the sample sizes of these categories.

The precision ratio is always contradictory with the recall ratio. The recall ratio is low when the precision ratio is high and vice versa. F-score is a measurement balancing the precision ratio and recall ratio. It is defined as the harmonic average of precision ratio and recall ratio which is adopted as the analysis method of our SVM recognition result.

\section{Experimental set up and procedures}

\subsection{Experimental set up}

As shown in Figure 1, the FDM machine used in our experiment is D-force delta bot and the material is PLA. The AE system involves the AE sensor, preamplifier and data acquisition system. The AE sensor used is $W S \alpha$ made by PAC with a working frequency band of [100 $\mathrm{kHz}, 1000 \mathrm{kHz}]$. The preamplifier is $2 / 4 / 6 \mathrm{C}$ made by PAC also with a working frequency range of $10-1200$ KHZ. Magnification of both $1 \mathrm{~dB}$ and $10 \mathrm{~dB}$ are optional. Data acquisition card chosen is ADLink DAQ-2100 which was embedded to the host of PC early. The software of signal acquisition is written in Labview. The sampling rate is adjustable. 


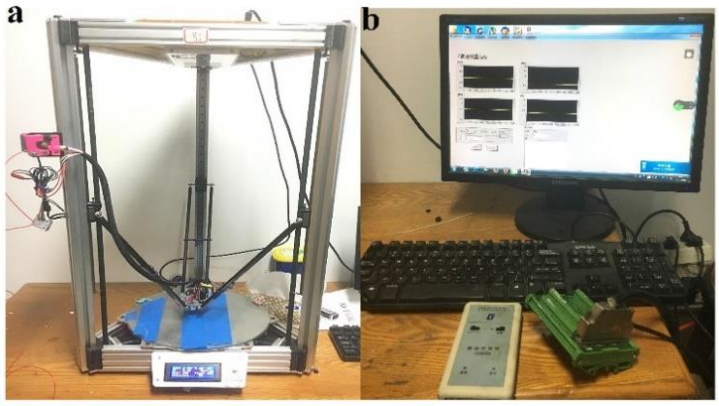

Figure 1. Experimental equipment used: (a) D-force delta bot FDM machine and (b) Signal acquisition system.

\subsection{Procedures}

The AE sensor was attached to the platform tightly using the vacuum grease in a distance of about $15 \mathrm{~cm}$ like Figure 2.

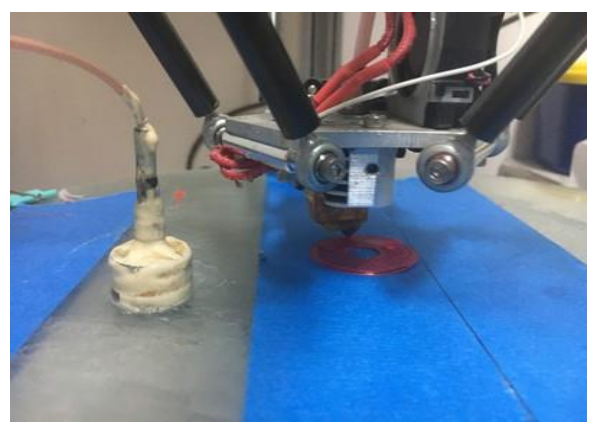

Figure 2. Location of AE sensor.

The printed part is a hollow cylinder and the g-code was generated by Cura. Process parameters set is shown in Table 1.

Table 1. The values of process parameters.

\begin{tabular}{|c|c|}
\hline Process parameters & Values \\
\hline Layer height & $0.15 \mathrm{~mm}$ \\
\hline Extruded temperature & $207^{\circ} \mathrm{C}$ \\
\hline Hotbed temperature & $45^{\circ} \mathrm{C}$ \\
\hline Filling density & $30 \%$ \\
\hline Filling speed & $30 \mathrm{~mm} / \mathrm{min}$ \\
\hline
\end{tabular}

60 groups of data samples were acquired respectively when the printing part was in normal, looseness and curl states with 300000 data contained in each group. 20 groups of data samples were used to train the HSMM and 10 groups of data samples were used to test the HSMM. The last 30 groups of data were samples under test.

\section{Results and discussions}

\subsection{Results of feature extraction}

To de-noise the acquired $\mathrm{AE}$ signals, fast Fourier transform (FFT) was carried out and $450 \mathrm{kHz}$ was chosen to be the cut-off frequency of low-pass filtering. And then, the signals were normalized by subtracting its mean value to be symmetrical about the zero axis. To relieve the computing burden of CPU, each groups of signals were divided into 10 equal frames. We use $T$ to represent the length of each frame data and $O^{k}=\left(O_{1}^{k}, \cdots, O_{k}^{T}\right)$ to represent the observation value of the $k$-th frame data. EEMD was carried out for each frame data and 13 IMFs were obtained.

The correlation coefficients between each IMF and the corresponding original signal were calculated and the standard deviations of them were calculated too which were the lower threshold when choosing the effective IMFs. Finally, the first, second, third and fourth IMF were selected as the effective IMFs. For each segmented signal, the energy of each effective IMF was calculated as the elements of a signal feature vector.

\subsection{Results of status recognition based on hidden semi-Markov model}

Left to right HSMM with 3 hidden states was employed. Matrix $\boldsymbol{\pi}$ is:

$$
\boldsymbol{\pi}=\left[\begin{array}{lll}
1 & 0 & 0
\end{array}\right]
$$

Matrix A owning the same state transition probability is like:

$$
\boldsymbol{A}=\left[\begin{array}{ccc}
0.5 & 0.5 & 0 \\
0 & 0.5 & 0.5 \\
0 & 0 & 1
\end{array}\right]
$$

The distribution of observed value represented as matrix $\mathbf{B}$ is Gaussian mixture distribution and the probability density function of dwell time in each state represented as $P_{i}(d)$ is single-Gaussian function. Kmeans was employed to the initialization process.

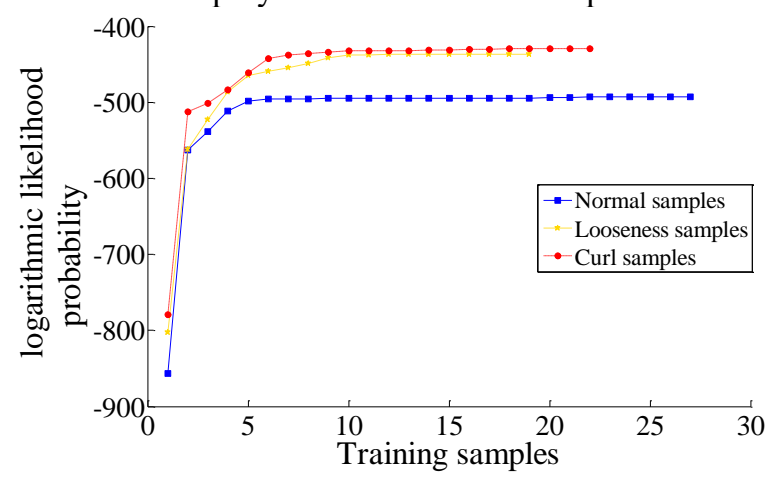

Figure 3. Training plot of HSMMs.

The number of maximal iteration and convergence error is respectively set as 50 and 0.01. 20 groups of samples were treated as training data. All three models 
converged within 30 iterations. The training plot of HSMMs constructed for the recognition of each state is shown in Figure 3. As we can see, the curve of each state tends to converge which proves the learning ability of HSMM.

After the training, 10 groups samples were treated as the test data. One example of the test plot based on the HSMM constructed for the recognition of normal state is shown in Figure 4. The recognized state is the one which owns the maximal logarithmic likelihood probability.

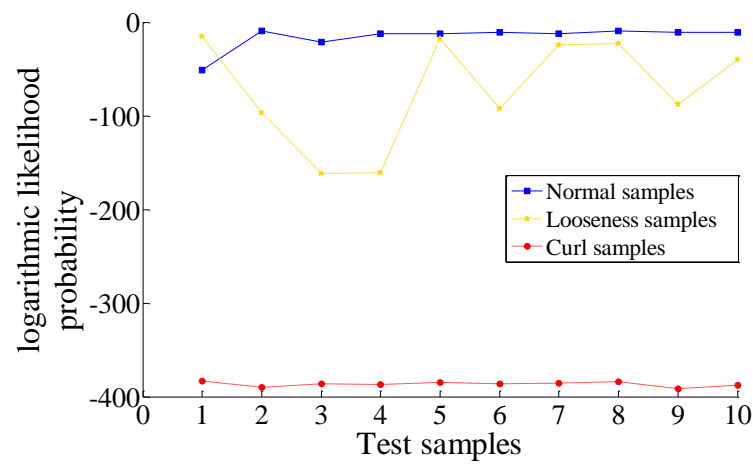

Figure 4. Test plot based on the HSMM constructed for the recognition of normal state.

Since a good result was obtained, the last 30 groups of samples were used in the states recognition process. The recognized results are shown in Table 2.

Table 2. Recognized results based on HSMM.

(a) Recognized result based on the HSMM constructed for the recognition of normal state.

\begin{tabular}{ccc}
\hline & \multicolumn{2}{c}{ Recognized result } \\
\cline { 2 - 3 } Actual state & Normal state & Other state \\
\hline Normal state & 29 & 1 \\
\hline Other state & 2 & 58 \\
\hline
\end{tabular}

(b) Recognized result based on the HSMM constructed for the recognition of loose state.

\begin{tabular}{ccc}
\hline & \multicolumn{2}{c}{ Recognized result } \\
\cline { 2 - 3 } Actual state & Looseness state & Other state \\
\hline Looseness state & 28 & 2 \\
\hline Other state & 1 & 59 \\
\hline
\end{tabular}

(c) Recognized result based on the HSMM constructed for the recognition of curling state.

\begin{tabular}{ccc}
\hline & \multicolumn{2}{c}{ Recognized result } \\
\cline { 2 - 3 } Actual state & Curl state & Other state \\
\hline Curl state & 30 & 0 \\
\hline Other state & 0 & 60 \\
\hline
\end{tabular}

\subsection{Results of status recognition based on support vector machine}

70 groups of samples were chosen as the training data while 30 groups of samples were chosen as the test data. Owing to the nature of a two class classifier, SVM was respectively constructed for the three states by training their corresponding samples. Each trained SVM was tested by samples of all three states respectively and the statistics results are shown in Table 3. One example of the test plot based on the SVM constructed for the recognition of normal state is shown in Figure 5. The non-linear kernel function was selected in the SVM constructed for the recognition of normal state while the linear function was employed in the SVM built for the recognition of looseness and curl states.

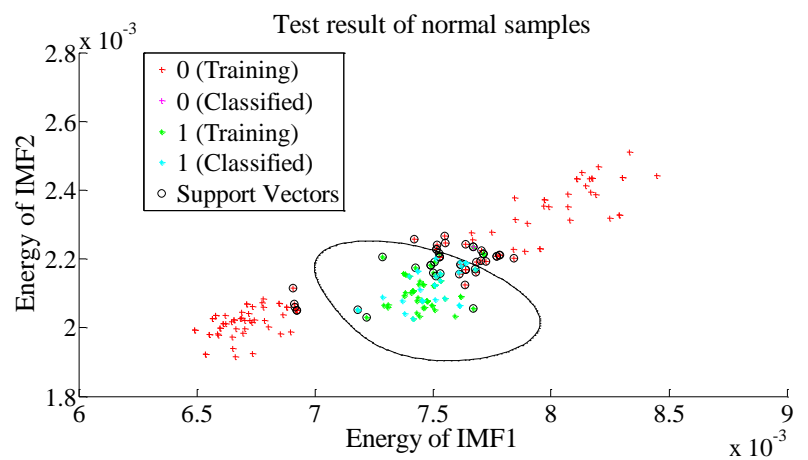

Figure 5. Plot of testing normal samples based on the SVM constructed for the recognition of normal state.

Table 3. Recognized results based on SVM.

(a) Recognized result based on the SVM constructed for the recognition of normal state.

\begin{tabular}{ccc}
\hline & \multicolumn{2}{c}{ Recognized result } \\
\cline { 2 - 3 } Actual state & Normal state & Other state \\
\hline Normal state & 29 & 1 \\
\hline Other state & 1 & 59 \\
\hline
\end{tabular}

(b) Recognized result based on the SVM constructed for the recognition of looseness state.

\begin{tabular}{ccc}
\hline & \multicolumn{2}{c}{ Recognized result } \\
\cline { 2 - 3 } Actual state & Looseness state & Other state \\
\hline Looseness state & 30 & 0 \\
\hline Other state & 8 & 52 \\
\hline
\end{tabular}


(c) Recognized result based on the SVM constructed for the recognition of curling state.

\begin{tabular}{ccc}
\hline & \multicolumn{2}{c}{ Recognized result } \\
\cline { 2 - 3 } Actual state & Curl state & Other state \\
\hline Curl state & 30 & 0 \\
\hline Other state & 0 & 60 \\
\hline
\end{tabular}

\subsection{Results of model performance measurement}

Values of $\mathrm{P}$ and $\mathrm{R}$ calculated for different models are shown in Table 4.

Table 4. $P$ and $R$ values.

(a) P and R of HSMMs constructed for the recognition of each state.

\begin{tabular}{ccccc}
\hline P and R & Normal & Looseness & Curl & Mean \\
\hline $\mathrm{P}$ & 0.9355 & 0.9655 & 1 & 0.9667 \\
\hline $\mathrm{R}$ & 0.9667 & 0.9333 & 1 & 0.9667 \\
\hline
\end{tabular}

(b) P and R of SVMs constructed for the recognition of each state.

\begin{tabular}{ccccc}
\hline P and R & Normal & Looseness & Curl & Mean \\
\hline $\mathrm{P}$ & 0.9667 & 0.7895 & 1 & 0.9187 \\
\hline $\mathrm{R}$ & 0.9667 & 1 & 1 & 0.9889 \\
\hline
\end{tabular}

Values of $F$ - score based on HSMM and SVM are calculated as:

$$
\begin{aligned}
& F-\operatorname{score}(\mathrm{HSMM})=\frac{2 \times \bar{P} \times \bar{R}}{\bar{P}+\bar{R}}=0.9668 \\
& F-\operatorname{score}(\mathrm{SVM})=\frac{2 \times \bar{P} \times \bar{R}}{\bar{P}+\bar{R}}=0.9525
\end{aligned}
$$

Thus, the $F$ - score value of HSMM is a bit higher than which of SVM.

The number of $k(k-1) / 2$ or $k$ SVMs need to be constructed for the multi-state classification problem due to it being a two class classifier which complicates the calculated process while HSMM is more appropriate to this problem. Further, HSMM is able to predict the development of defects according to signs it appeared.

\section{Conclusion}

AE sensor was applied in our article. The feature extraction method EEMD and pattern recognition methods of HSMM and SVM were employed to the status recognition of the printing part. The $F$ - score value was calculated to evaluate the performance of each constructed model. The results show that above methods are able to recognised the normal, looseness and curl states and the recognition ability of HSMM is a bit higher than SVM.

\section{References}

1. Y. A. Jin, Y. He, G. H. Xue, et al. Int. J. Adv. Manuf. Technol., 77(5-8):927-937 (2015).

2. A. K. Sood, R. K. Ohdar, S. S. Mahapatra. Journal of Advanced Research, 3(1):81-90 (2012).

3. A. Peng, X. Xiao, R. Yue. Int. J. Adv. Manuf. Technol, 73(1-4):87-100 (2014).

4. A. Huang, J. Lee. Rapid Prototyping J., 19(4):291299 (2013).

5. A. K. Sood, A. Equbal, V. Toppo, et al. CIRP AnnManuf. Technol., 5(1):48-54 (2012).

6. M. R. K. Ravari, M. Kadkhodaei, M. Badrossamay, et al. Int. J. Mech. Sci., 88:154-161 (2014).

7. M. Abdelrahman, T. L. Starr. Int. J. Adv. Manuf. Technol., 84(5-8):831-842 (2016).

8. P. Rao, J. Liu, D. Roberson, et al. J. Manuf. Sci. Eng.-Trans. ASME, 137(6):1007-1 - 1007-12 (2015).

9. M. M. Kirka, R. Dinwiddie. Understanding the thermal sciences in the electron beam melting process through in-situ process monitoring[C]// Society of Photo-Optical Instrumentation Engineers. Society of Photo-Optical Instrumentation Engineers (SPIE) Conference Series, 1016900 (2017).

10. B. Lane, S. Moylan, E. Whitenton, et al. Rapid Prototyping J, 22(5):778-787 (2016).

11. C. Kousiatza, D. Karalekas. Mater. Des., 97: 400406 (2016).

12. A. Kantaros, D. Karalekas. Mater. Des., 50: 44-50 (2013).

13. W. Yi, H. Ketai, Z. Xiaomin, et al. Machine vision based statistical process control in fused deposition modeling[C]//Industrial Electronics and Applications (ICIEA), 2017 12th IEEE Conference on. IEEE, 936-941 (2017).

14. E. A. C. Koskelo, E. B. Flynn. Scanning laser ultrasound and wavenumber spectroscopy for inprocess inspection of additively manufactured parts $[\mathrm{C}] / /$ Nondestructive Characterization and Monitoring of Advanced Materials, Aerospace, and Civil Infrastructure 2016. International Society for Optics and Photonics, 9804: 980418 (2016).

15. I. Cummings, E. Hillstrom, R. Newton, et al. Inprocess ultrasonic inspection of additive manufactured parts[M]//Topics in Modal Analysis \& Testing, Volume 10. Springer, Cham, 235-247 (2016).

16. I. T. Cummings, M. E. Bax, I. J. Fuller, et al. A Framework for Additive Manufacturing Process Monitoring \& Control[M]//Topics in Modal Analysis \& Testing, Volume 10. Springer, Cham, 137-146 (2017).

17. Z. Wu, N. E. Huang. Advances in adaptive data analysis, 1(01): 1-41 (2009).

18. X. Zhang, Y. Liang, J. Zhou. Measurement, 69: 164179 (2015).

19. A. Tabrizi, L. Garibaldi, A. Fasana, et al. Meccanica, 50(3): 865-874 (2015). 
20. X. Xue, J. Zhou, Y. Xu, et al. Mech. Syst. Signal Proc., 62: 444-459 (2015).)

21. L. Meng, J. Xiang, Wang Y, et al. Mech. Syst. Signal Proc., 50: 101-115 (2015).

22. X. Chen, G. Cheng, X. Shan, et al. Measurement, 73: 55-67 (2015).

23. S. Z. Yu. Artif. Intell., 174(2): 215-243(2010).

24. M. Dong, D. He. Mech. Syst. Signal Proc., 21(5): 2248-2266 (2007).

25. O. Geramifard, J. X. Xu, J. H. Zhou, et al. IEEE Trans. Ind. Inform., 8(4): 964-973 (2012).

26. H. Zen, K. Tokuda, T. Masuko, et al. IEICE Trans. Inf. Syst., 90(5): 825-834 (2007).

27. V. Vapnik. The nature of statistical learning theory[M]. Springer science \& business media (2013).

28. R. B. Chinnam. Int. J. Prod. Res., 40(17): 4449-4466 (2002).

29. A. Widodo, B. S. Yang. Mech. Syst. Signal Proc., 21(6): 2560-2574 (2007).

30. J. Sun, M. Rahman, Y. S. Wong, et al. Int. J. Mach. Tools Manuf., 44(11): 1179-1187 (2004).

31. Z. Yao, D. Mei, Z. Chen. J. Mater. Process. Technol., 210(5): 713-719 (2010).

32. J. Friedman. Another approach to polychotomous classification[R]. Technical report, Department of Statistics, Stanford University (1996). 